\title{
Tratamento de leucemia linfói de aguda e crescimento
}

\author{
I.M.U. Monteiro, A.J . Bedone, C.L.B. Pinto, S.R. Brandalise \\ Área de Ginecologia do Departamento de Tocoginecologia da Faculdade de Cências Médicas - Centro de Atenção Integral à Saúde \\ da Mulher da UNICAMP, Campinas, SP.
}

RESUMO - ОвJ etıvo. Determinar alterações no crescimento após o tratamento de leucemia linfóide aguda em meninas.

Pacientes e Método. Realizou-se estudo retrospectivo com 59 meninas que apresentavam medi das de estatura antes e com no mínimo um ano do tratamento, subdivididas de acordo com a dose de radioterapia cranial utilizada [18 ou 24 Grays (Gy)] e com a idade no início do tratamento (antes e após os cinco anos de idade).

Resultados. Observou-se deficiência do crescimento com um, dois e mais de dois anos do trata-

\section{INTRODUÇÃO}

Durante o tratamento de leucemi a linfói de aguda (LLA), vários aspectos têm si do apontados como causadores de alterações no crescimento. Entre estes, pode-se citar a própria doença, a nutrição deficiente, a ocorrência de infecções, a quimioterapia e a radioterapia cranial. A somatória de todos esses fatores determina uma desaceleração do crescimento ${ }^{1}$.

Parece haver consenso que a radioterapia é o principal causador dessas alterações, por levar a uma diminuição de secreção do hor mônio de crescimento $(\mathrm{GH})^{2-6}$. A deficiência de $\mathrm{GH}$ pode ser parcial e persistir vári os anos após o tratamento. A manifestação dessa deficiência depende do estádio puberal em que a criança está sendo observada. Na fase pré-puberal são suficientes pequenas quantidades de GH para assegurar o crescimento normal; nessa fase, os efeitos nem sempre são bem dimensionados. Entretanto, quando a criança atinge a puberdade, o aumento da demanda de GH não é suprido, tornando mais evidentes as alterações do crescimento, com achatamento do estirão puberal ${ }^{7}$.

A dose de radioterapia parece ter importância nas repercussões futuras sobre o crescimento. A comparação entre crianças tratadas com 18 ou 24Gy evidenciou que o primeiro grupo apresentou recuperação completa da curva de crescimento ${ }^{8}$. Entretanto, há estudos que mostraram alterações persistentes do crescimento, mesmo com essas doses. Observou-se deficiência de GH em crianças com mento. 0 crescimento foi mais afetado no grupo tratado com 24Gy, quando comparado com 18Gy. Não houve diferenças com relação à idade no início do tratamento.

ConCLUSÕEs. Houve retardo no cresci mento após o tratamento, independentemente da idade em que foi realizado, mas dependente da dose de radioterapia cranial utilizada.

UNITERMOS: Crescimento. Leucemia linfóide aguda - indicações.

doses de 18Gy9-11, permanecendo, portanto, algumas controvérsias a respeito dos efeitos dessa dose de radioterapia sobre o crescimento.

O aumento nas taxas de sobrevida e as novas possibilidades de cura de LLA têm permitido que um maior número de sobrevi ventes chegue a idade adulta. O crescimento pode ser retardado pela radioterapia, e tanto a dose de radioterapia como a idade em que o tratamento é realizado podem ter influência na estatura final.

E ste trabal ho teve como obj etivo avaliar a influência da idade por ocasião do diagnóstico e a dose de radioterapia cranial utilizada.

\section{PACIENTES E MÉTODO}

F oi realizado um estudo retrospecti vo no Centro Infantil de Investigações Hematológicas “Dr. Domingos A. Boldrini" - Campinas, com meninas tratadas de LLA, no período de janeiro de 1980 a janeiro de 1991.

E $m$ todas as meninas, o tratamento foi realizado antes da puberdade e incluiu quimioterapia sistêmica e intratecal, além de radioterapia cranial. Foram selecionadas 59 pacientes que apresentavam estatura medida no início e com um ano após o tratamento. Dentre as 59 pacientes, 51 tinham também anotadas as medi das de estatura com dois anos após o tratamento. Das 51 pacientes, 36 tinham avaliação da estatura com mais de dois anos do tratamento. O tempo médio de acompanhamento dessas pacientes foi 5,3 ( $D P=1,8$ ano). As pacien- 
tes foram subdivididas de acordo com a dose de radioterapia cranial utilizada (18 ou 24Gy) e de acordo com a idade de início do tratamento, antes dos cinco e com cinco ou mais anos de idade.

A estatura foi expressa com escore de desviopadrão (SDS) obtido mediante a seguinte fórmula:

$$
\frac{x-x}{D P}
$$

onde $x$ é a estatura em centímetros, $x$ a estatura média esperada para a idade e DP o desvio-padrão da estatura correspondente nesta idade.

Foram utilizados os testes t de Student e a regressão linear múltipla para a análise estatística, considerando-se estatisticamente significativas quando $\mathrm{p}<0,05$.

\section{RESULTADOS}

A comparação das médias dos SDS das estaturas, antes do tratamento e em diferentes épocas após o tratamento, mostrou desacel eração do cresci mento (tabela 1).

Quando comparadas as médias dos SDS da estatura ao final do tratamento e com um e dois anos após, observou-se aumento da média tanto com um quanto com dois anos, denotando a recuperação do crescimento (tabela 2).

A observação isol ada do grupo submetido a tratamento com 18Gy mostrou diminuição da estatura nos vários períodos de observação, em relação ao início do tratamento (tabela 3).

Resultados semel hantes for am observados com o grupo tratado com 24Gy (tabela 4).

A análise em meninas tratadas antes dos cinco anos de idade mostrou di minuição das médias com um e dois anos após o tratamento (tabela 5).

Com relação ao grupo tratado com cinco ou mais anos de idade, observou-se diminuição das médias dos SDS da estatura em todos os períodos observados, em comparação ao início do tratamento (tabela 6).

Considerando-se como variáveis independentes a idade no início do tratamento e a dose de radioterapia cranial, realizou-se a análise de regressãolinear múltipla para a avaliação dos fatores associados à variação do SDS durante o período de observação (SDS da última avaliação, subtraído o SDS inicial).

Observou-se que a dose de radioterapia (24Gy) esteve associada com mai or deficiência do crescimento (tabela 7).

\section{DISCUSSÃO}

Nossos resultados evidenciaram deficiência do crescimento nas várias avaliações feitas após o
Tabela 1 - Comparação das médias dos SDS da estatura segundo o tempo em relação ao início do tratamento

\begin{tabular}{|lcccc|}
\hline \multicolumn{1}{|c|}{ Variável } & $\begin{array}{c}\text { Média } \\
\text { SDS }\end{array}$ & $\begin{array}{c}\text { Desvio-padrão } \\
\text { SDS }\end{array}$ & $\mathbf{n}$ & $\mathbf{p}^{*}$ \\
SDS inicial & 0,09 & 0,93 & 59 & $<0,001$ \\
SDS 1 ano & $-0,35$ & 0,89 & 59 & \\
SDS inicial & 0,13 & 0,93 & 51 & $<0,001$ \\
SDS 2 anos & $-0,30$ & 0,95 & 51 & \\
SDS inicial & 0,28 & 0,93 & 36 & $<0,002$ \\
SDS $>2$ anos & $-0,33$ & 1,05 & 36 & \\
\hline * Teste $t$ de Student para amostras emparelhadas. & \\
SDS $=$ escore de desvio padrão.
\end{tabular}

Tabela 2 - Comparação das médias dos SDS da estatura segundo o tempo relacionado ao tratamento

\begin{tabular}{|lcccc|}
\hline \multicolumn{1}{|c}{ Variável } & $\begin{array}{c}\text { Média } \\
\text { SDS }\end{array}$ & $\begin{array}{c}\text { Desvio-padrão } \\
\text { SDS }\end{array}$ & $\mathbf{n}$ & $\mathbf{p}^{*}$ \\
SDS final do tratamento & $-0,46$ & 0,80 & 59 & $<0,02$ \\
SDS 1 ano & $-0,35$ & 0,89 & 59 & \\
SDS final do tratamento & $-0,49$ & 0,80 & 51 & $<0,01$ \\
SDS 2 anos & $-0,30$ & 0,95 & 51 & \\
SDS final do tratamento & $-0,44$ & 0,78 & 36 & n.s. \\
SDS $>$ 2 anos & $-0,33$ & 1,05 & 36 & \\
\hline * Teste $t$ de Student para amostras emparelhadas. & & \\
SDS = escore de desvio-padrão; n.s. = não-significativo. \\
\hline
\end{tabular}

Tabela 3 - Comparação das médias dos SDS no grupo tratado com dose de radioterapia cranial de $18 \mathrm{~Gy}$

\begin{tabular}{|lcccc|}
\hline \multicolumn{1}{|c}{ Variável } & $\begin{array}{c}\text { Média } \\
\text { SDS }\end{array}$ & $\begin{array}{c}\text { Desvio-padrão } \\
\text { SDS }\end{array}$ & $\mathbf{n}$ & $\mathbf{p}^{*}$ \\
SDS inicial & $-0,07$ & 0,92 & 46 & $<0,001$ \\
SDS 1 ano & $-0,42$ & 0,86 & 46 & \\
SDS inicial & $-0,05$ & 0,91 & 38 & $<0,01$ \\
SDS 2 anos & $-0,41$ & 0,87 & 38 & \\
SDS inicial & 0,06 & 0,96 & 25 & $<0,05$ \\
SDS $>2$ anos & $-0,31$ & 1,03 & 25 & \\
\hline * Teste $t$ de Student para amostras emparelhadas. & \\
SDS = escore de desvio-padrão. \\
\hline
\end{tabular}

Tabela 4 - Comparação das médias dos SDS no grupo tratado com 24Gy

\begin{tabular}{|lcccc|}
\hline \multicolumn{1}{|c}{ Variável } & $\begin{array}{c}\text { Média } \\
\text { SDS }\end{array}$ & $\begin{array}{c}\text { Desvio-padrão } \\
\text { SDS }\end{array}$ & $\mathbf{n}$ & $\mathbf{p}^{*}$ \\
SDS inicial & 0,67 & 0,77 & 13 & $<0,01$ \\
SDS 1 ano & $-0,10$ & 1,02 & 13 & \\
SDS inicial & 0,67 & 0,77 & 13 & $<0,05$ \\
SDS 2 anos & 0,02 & 1,12 & 13 & \\
SDS inicial & 0,78 & 0,65 & 11 & $<0,05$ \\
SDS $>2$ anos & $-0,37$ & 1,15 & 11 & \\
\hline * Teste $t$ de Student para amostras emparelhadas. \\
SDS = escore de desvio-padrão. \\
\hline
\end{tabular}




\begin{tabular}{|c|c|c|c|c|}
\hline \multicolumn{5}{|c|}{$\begin{array}{l}\text { Tabela } 5 \text { - Comparação das médias dos SDS em menina } \\
\text { tratadas antes dos cinco anos de idade }\end{array}$} \\
\hline \multicolumn{5}{|c|}{$\begin{array}{l}\text { Média } \\
\text { SDS }\end{array}$} \\
\hline $\begin{array}{l}\text { SDS inicial } \\
\text { SDS } 1 \text { ano }\end{array}$ & $\begin{array}{r}0,41 \\
-0,02\end{array}$ & $\begin{array}{l}0,96 \\
0,91\end{array}$ & $\begin{array}{l}29 \\
29\end{array}$ & $<0,001$ \\
\hline $\begin{array}{l}\text { SDS inicial } \\
\text { SDS } 2 \text { anos }\end{array}$ & $\begin{array}{l}0,49 \\
0,09\end{array}$ & $\begin{array}{l}0,98 \\
0,98\end{array}$ & $\begin{array}{l}25 \\
25\end{array}$ & $<0,02$ \\
\hline $\begin{array}{l}\text { SDS inicial } \\
\text { SDS > } 2 \text { anos }\end{array}$ & $\begin{array}{l}0,61 \\
0,08\end{array}$ & $\begin{array}{l}0,94 \\
1,03\end{array}$ & $\begin{array}{l}19 \\
19\end{array}$ & n.s. \\
\hline
\end{tabular}

Tabela 6 - Comparação das médias dos SDS em meninas tratadas com cinco ou mais anos de idade

\begin{tabular}{|lcccc|}
\hline \multicolumn{1}{|c}{ Variável } & $\begin{array}{c}\text { Média } \\
\text { SDS }\end{array}$ & $\begin{array}{c}\text { Desvio-padrão } \\
\text { SDS }\end{array}$ & $\mathbf{n}$ & $\mathbf{p}^{*}$ \\
SDS inicial & $-0,21$ & 0,81 & 30 & $<0,01$ \\
SDS 1 ano & $-0,66$ & 0,77 & 30 & \\
SDS inicial & 0,20 & 0,74 & 26 & $<0,001$ \\
SDS 2 anos & $-0,66$ & 0,75 & 26 & \\
SDS inicial & $-0,09$ & 0,79 & 17 & $<0,02$ \\
SDS $>2$ anos & $-0,80$ & 0,88 & 17 & \\
\hline * Teste $t$ de Student para amostras emparelhadas. \\
SDS $=$ escore de desvio-padrão. \\
\hline
\end{tabular}

Tabela 7 - Fatores associados à variação do SDS durante todo o período de observação: regressão linear múltipla

\begin{tabular}{|lccc|}
\hline Variável & Coeficiente & E.P. coeficiente & $\mathbf{p}$ \\
Dose de radioterapia & $-0,6562$ & 0,2578 & 0,0137 \\
Constante & $-0,2884$ & 0,1210 & 0,0205 \\
\hline Variáveis independentes analisadas: & & \\
- dose de radioterapia (18 ou 24Gy) & \\
- idade no início do tratamento & \\
SDS = escore de desvio-padrão.
\end{tabular}

término do tratamento. $\mathrm{E} m$ todos os períodos analisados, as meninas do grupo tratado estiveram abaixo da média das meninas normais. A deficiência do crescimento foi persistente, pois, anos após o término do tratamento, as meninas estavam mais baixas em relação à população geral, fato não observado no início do tratamento.

O uso da radioterapia cranial tem sido apontado como a causa dessa deficiência persistente, por diminuir a secreção de hormônio do crescimento $(\mathrm{GH})^{4}$.

$O$ efeito persistente da radi oter apia sobre o cresci mento já havia sido observado em um estudo com dez meninas submetidas a tratamento radioterápi $\mathrm{Co}^{12}$. Outro estudo comprovou esse efeito persistente da radioterapia ao demonstrar que meninas submetidas apenas a tratamento para LLA com quimioterápicos apresentaram recuperação do crescimento após cessado o tratamento ${ }^{13}$.

Ao que parece, o efeito da radioterapia é dose dependente. E m estudo realizado por Cicognani et al. ${ }^{8}$, observou-se que o grupo de meni nas submetido a 18Gy apresentou recuperação completa do crescimento após dois anos do tratamento, ao contrário do grupo submetido a 24Gy. Em nosso estudo, os resultados mostraram deficiência do crescimento após o término do tratamento, nos dois grupos. As diferenças entre este estudo e o de Cicognani et al. ${ }^{8}$ podem ser explicadas pelo maior número de pacientes submetidas a $18 \mathrm{~Gy}$ e pelo mai or tempo de acompanhamento em nosso estudo.

Outro estudo corroborou os resultados por nós encontrados. A avaliação de 89 meninas (42 tratadas com 24Gy e 47 com 18Gy) mostrou resultados semel hantes aos nossos ${ }^{6}$. Deve-se ressaltar, no entanto, que em nosso estudo o crescimento foi mais afetado pelo uso de 24Gy.

As discussões sobre a influência da idade no início do tratamento dizem respeito à adequação da secreção do GH à i dade da menina. A necessi dade de GH durante a infância é menor que no período prépuberal. Seria de se esperar, portanto, que as manifestações da deficiência de GH fossem mais evidentes próximas à puberdade. Não se pode esquecer, por outro lado, que o efeito da radioterapia sobre a secreção de GH é persistente; uma criança, tratada nos primeiros anos de vida, poderia apresentar um comprometimento importante de seu crescimento, pois a defi ciência de GH sefaria sentir por mais tempo.

N ossos resultados não mostraram diferença com relação à idade por ocasião do tratamento. Entretanto, qualquer conclusão definitiva a esse respeito poderá ser precipitada. O número de crianças tratadas de LLA em nosso e em outros estudos não permite que se estabeleça, com segurança, se há relação entre a faixa etária e a deficiência de crescimento.

\section{CONCLUSÕES}

As médias das estaturas foram menores ao final do acompanhamento do que no início, principalmente no grupo tratado com 24Gy. Não houve influência da idade por ocasião do tratamento.

\section{SUMMARY}

\section{Acute lymphocytic leukaemia treatment and growth}

BACKGROUND. The treatment of the acutelymphocytic leukaemia can detrmine impaired growth. 
Subj eCts And Method. All thepatients had length measurements at the time of the beginning of the treatment and, at least, one year after the end of it.

ConcLusions. There was impaired growth after the treatment according to the dose regimens (18 or 24 Grays). No relation was observed related to the age at the diagnosis. [Rev Ass Med Brasil 1998; 44(2): 77-80.]

KEY WORDS: Growth. Acute lymphocytic leukaemia treatment.

\section{REFERÊNCIAS BIBLIOGRÁFICAS}

1. Monteiro IMU. Tratamento da leucemia linfóide aguda em meninas: repercussões sobre o desenvolvimento puberal e o crescimento. Dissertação de Mestrado. Unicamp, 1994.

2. Perry-Keene DA, Connely J F, Young RA, Wettenhall HNB, Martin FIR. Hypothalamic-hypopituitarism following external radiotherapy for tumours distant from the adenohypophisis. Clin Endocrinol 1976; 5: 373-80.

3. Oliff A, Bode V, Bercu BB et al. Hypothalamic-pituitary disfunction following CNS prophylaxis in acute lymphocytic leukemia: correlation with CT scan abnormalities. Med Pediatr Oncol 1979; 7: 141-51.

4. Wells R, Foster M, D'ercole AJ , McMillan C. The impact of cranial irradiation on the growth of children with acute Iymphocytic leukemia. Am J Dis Child 1983; 137:37-9.
5. Leiper AD, Stanhope R, Preece MA, Grant DB, Chessels J M. Precocious or early puberty and growth failure in girls treated for acute lymphoblastic leukaemia. Horm Res 1988; 30: 72-6.

6. Uruena M, Stanhope R, Chessells J M, Leiper AD. Impaired pubertal growth in acute lymphoblastic leukaemia. Arch Dis Child 1991; 66:1403-7.

7. Moell C. Disturbed pubertal growth in girls after acute leukaemia: a relative growth hormone insufficiency with late presentation. Acta Paediatr Scand 1988; 343:162-6.

8. Cicognani A, Cacciari $E$, Vecchi $V$ et al. Differential effects of 18- and 24-Gy cranial irradiation on growth rate and growth hormone release in children with prolonged survival after acute Iymphocytic leukemia. Am J Dis Child 1988; 142: 1.199-202.

9. Kirk J A, Raghupathy $P$, Stevens MM. Growth failure and growth-hormone deficiency after treatment for acute lymphoblastic leukaemia. Lancet 1987; 1:190-3.

10. Clayton PE, Shalet S, Morris-J ones PH, Price DA. Growth in children treated for acute Iymphoblastic leukemia. Lancet 1988; 1: 460-2.

11. Brauner R, Thibaud E, Rappaport R. Fonction gonadique après traitement des cancers de l'enfant. Ann Pediatr 1990; 37: 437-43.

12. Moell C, Garwicz S, Westgren U, Wieb T, Albertsson-Wikland K. Blunted pubertal growth after leukemia: a new pattern of growth hormone insuficiency. Horm Res 1988; 30: 68-71.

13. Katz J A, Chambers B, Everhart C, Marks J F, Buchanan GR. Linear growth in children with acute lymphoblastic leukemia treated without cranial irradiation. J Pediatrics 1991; 118: $575-8$. 